\title{
Robotic Transthoracic First Rib Resection for Neurogenic Thoracic Outlet Syndrome
}

\author{
Farid Gharagozloo*, Mark Meyer \\ Center for Advanced Thoracic Surgery, Advent Health Celebration, University of Central Florida, Celebration, USA \\ Email: *Gharagozloof@aol.com
}

How to cite this paper: Gharagozloo, F. and Meyer, M. (2022) Robotic Transthoracic First Rib Resection for Neurogenic Thoracic Outlet Syndrome. World Journal of Cardiovascular Surgery, 12, 1-11. https://doi.org/10.4236/wjcs.2022.121001

Received: January 1, 2022

Accepted: January 27, 2022

Published: January 30, 2022

Copyright $\odot 2022$ by author(s) and Scientific Research Publishing Inc. This work is licensed under the Creative Commons Attribution International License (CC BY 4.0).

http://creativecommons.org/licenses/by/4.0/

\begin{abstract}
Purpose: Neurogenic Thoracic Outlet Syndrome accounts for over 95\% of patients with TOS. We report a single institution experience with robotic first rib resection in patients with Neurogenic TOS. Methods: The diagnosis of NTOS was made in patients in whom all specific localizing and diagnostic orthopedic and neurologic conditions were ruled out. Preoperative diagnostic tests included a comprehensive history and physical exam, Chest X-ray, Chest CT, MRI if the cervical spine, Nerve conduction studies, and Magnetic Resonance angiography of the Thoracic outlet with arm maneuvers (MRA). Patients with NTOS who underwent robotic first rib resection with disarticulation of the costosternal joint and scalenectomy. Results: There were 137 patients ( 47 men and 90 women). Mean age was $34 \pm 9.5$ years. Operative time was 93 minutes \pm 10.3 minutes. There were no intraoperative complications. There was no injury to the subclavian vessels during the dissection. There were no neurovascular complications. There was no 30 or 90 day mortality. Quick DASH Scores (Mean \pm SEM) decreased from $60.3+/ 2.1$ preoperatively to $5 \pm 2.3$ in the immediate postoperative period, and 3.5+/1.1 at 6 months. (P $<0.01)$ Immediate relief of symptoms was seen in all patients (100\%). Complete relief of symptoms was seen in 133/137 (97\%) of patients. Conclusions: Robotic resection of the medial aspect of the first rib with disarticulation of the costo-sternal joint is associated with excellent relief of neurologic symptoms in patients with Neurogenic Thoracic Outlet Syndrome.
\end{abstract}

\section{Keywords}

Thoracic Outlet Syndrome, Neurogenic, Robotic Surgery, Minimally Invasive Surgery

\section{Introduction}

In 1956, the term "Thoracic Outlet Syndrome" (TOS) was first proposed for the 
purpose of unifying the symptoms associated with many upper extremity neurovascular compression syndromes [1]. Since that report, TOS has been classified based on symptoms, rather than the underlying pathology, with the subgroups of neurogenic (NTOS), venous (VTOS or PSS), and arterial (ATOS) TOS [2]. Neurogenic TOS accounts for over $95 \%$ of the cases, followed by venous ( $3 \%$ - 5\%) and arterial (1\% - 2\%) [3]. Only 5\% of patients with the diagnosis of neurogenic TOS are found to have a cervical rib or cervical bands and are best classified as cervical rib syndrome. The majority of patients with symptomatic NTOS have negative findings on objective studies, and therefore, are classified as Disputed NTOS. Ironically, in patients with DTOS surgery is often used as a last resort in order to establish the diagnosis. A study of patients with DTOS who had persistent upper extremity pain following first rib resection by the transaxillary and supraclavicular approaches, revealed persistent extrinsic compression of the subclavian innominate junction on dynamic MRA. These patients underwent video-assisted exploration of the chest, which showed a persistent costo-sternal joint despite evidence for prior removal of the first rib. Disarticulation of the cost-sternal joint and removal of the remaining portion of the first rib alleviated the extrinsic compression of the subclavian-innominate vein junction on postoperative dynamic MRA and resulted in relief of Neurogenic symptoms in all patients [4]. Based on this observation, it has been hypothesized that DTOS may be the manifestation of nerve pain which results from venous compression and the resultant venous ischemia of the nerves in the upper extremity.

First Rib resection has been advocated for the remaining patient with DTOS. Surgical approaches to resection of the first rib have included transthoracic, transaxillary, supraclavicular, infraclavicular, and thoracoscopy [5] [6]. However, these approaches are associated with neurovascular complications, incomplete decompression of the subclavian vein and the medial aspect of the thoracic outlet, and incomplete resection of the most medial portion of the rib. Robotic first rib resection has been shown to be feasible, safe, and associated with excellent outcomes in patients with Paget Schroetter Syndrome [7] [8]. We report a single institution experience with robotic first rib resection and scalenectomy in patients with Neurogenic TOS.

\section{Methods}

This is a retrospective cohort study using a prospective database from a consecutive series of patients from January 2010 to January 2021. Patients with NTOS who underwent robotic first rib resection with disarticulation of the costosternal joint and scalenectomy were reviewed. The diagnosis of NTOS was made in a smaller cohort of patients from a larger group who presented with pain, paranesthesia and other neurologic symptoms affecting the upper extremity, the ipsilateral neck or shoulder. In this cohort, all specific localizing and diagnostic orthopedic and neurologic conditions were ruled out. Preoperative diagnostic tests included a comprehensive history and physical exam, Chest X-ray, Chest 
CT, MRI if the cervical spine, Nerve conduction studies, and Magnetic Resonance angiography of the Thoracic outlet with arm maneuvers (MRA). Surgery was offered to patients if the MRA showed extrinsic compression of the neurovascular bundle in the thoracic outlet by the abnormal portion of the first rib at the costo-sternal junction.

Surgical technique:

The surgical technique has been described for patients with PSS [8]. The plan of the operation is to remove the medial portion of the first rib directly underlying the subclavian artery and vein and disarticulate the rib at the costo-sternal joint. The portion of the rib lateral to the subclavian artery and the costo-vertebral joint are left intact. The procedure is performed in 4 phases:

Phase I: General anesthesia with single lung ventilation is used and patients are placed in the lateral decubitus position with the affected side up (Figure 1). Three $2 \mathrm{~cm}$, nontrocar, incisions are made. In the right chest, Incision \#1 is made at the $7^{\text {th }}$ intercostal (IC) space at the midaxillary line. Incision $\# 2$ is made in the $4^{\text {th }}$ IC space at the anterior axillary line. Incision \#3 is made in the $7^{\text {th }}$ IC space at the posterior axillary line. A $1 \mathrm{~cm}$ incision (\#4) is made in the $8^{\text {th }}$ intercostal space at the anterior axillary line. The placement of incisions is in a mirror image configuration for the left chest. A retractor (Endopaddle Retract; Auto Suture, Covidien incorporated, Mansfield, MA) is introduced through this incision and used to retract the lung inferiorly. At the end of the procedure, a chest drain is inserted through this incision.

Phase II: The surgical robot (da Vinci Si, Intuitive Surgical, Inc., Sunnyvale, $\mathrm{CA}$ ) is positioned over the head of the patient. A 30 degree up viewing camera is placed in incision \#1 (Video 1). The right robotic arm with a hook cautery is positioned in incision \#2. The left robotic arm with a long tip grasper is positioned in incision \#3. The pleura overlying the first rib is dissected. The superior and inferior edges of the rib are clearly delineated and the costo-sternal joint is identified. The internal thoracic artery and vein and phrenic nerve are identified and preserved.

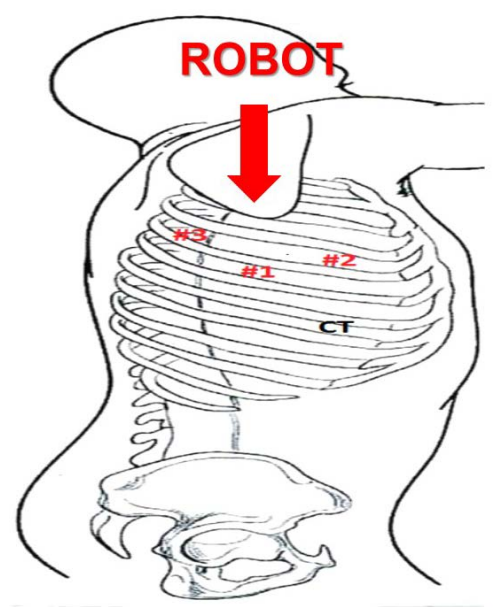

Figure 1. Patient positioning and port placement. 
Phase III: The robotic arms are withdrawn. The camera is introduced through incision \#2 and held by the assistant. A $6 \mathrm{~mm}$ thoracoscopic Kerrison bone cutter (Depuy Inc., Raynham, MA) is introduced through incision \#1, and the rib under the subclavian artery is divided. This area represents the thinnest portion of the first rib and is most suitable for an osteotomy. The division of the rib at its midpoint allows for the rib to be pivoted on the costo-sternal and costo-vertebral joints in a trap door configuration.

Phase IV: The robotic arms are replaced. A 30 degree down-viewing camera is placed in incision \#1. The right robotic arm with a hook cautery is positioned in incision \#2. The left robotic arm with a second hook cautery is positioned in incision \#3. By virtue of using two hook cautery instruments it is not possible to use the da Vinci internal cautery generator. Therefore, an external cautery generator (Valley Lab, Medtronic, North Haven, CT), set on 30 "Coag”, 30 "Cut", with a separate foot pedal is required. The hook in the left robotic arm is used to exert downward traction on the bone and the hook in the right robotic arm with cautery is used to divide the scalene muscles off of the first rib and disarticulate the rib at the costo-sternal joint.

A video of the procedure can be accessed at

https://www.youtube.com/watch?v=2mCKcgAAjb8\&t=9s (Video 1)

Assessment of Subjective symptoms:

Subjective symptoms were assessed by Disabilities of the Arm, Shoulder and Hand Questionnaire Score (QuickDASH), preoperatively and postoperatively in the immediate postoperative period and 6 months. The QuickDASH is an abbreviated version of the original DASH outcome measure. In comparison to the original 30-item DASH outcome measure, the QuickDASH only contains 11 items. It is a questionnaire that measures an individual's ability to complete tasks, absorb forces, and severity of symptoms. The QuickDASH tool uses a 5-point Likert scale from which the patient can select an appropriate number corresponding to his/her severity level/function level [9] [10].

Data Analysis:

Data were exported from Excel (Microsoft Corp., Redmond, WA.). Information was obtained through hospital databases, medical records, and our prospective database. The data was prospectively accrued and retrospectively analyzed. Individual consent was waived for inclusion in this study; however, it was required and obtained to enter patient data in the prospective database. This study was reviewed institutional review board and determined to be exempt under 45 CFR 46.101 (b).

Data points analyzed included indication for operation, patient age and sex, operative time, morbidity, death, preoperative and postoperative Quick Dash scores. The demographic data was reported as Mean \pm SD. Follow-up was performed by physical exam, and MRA with maneuvers at 1 month in all patients. At the time of follow up, the success of the first rib resection was determined by the complete resolution of symptoms and demonstration of the relief of extrinsic compression of the subclavian innominate junction on the MRA. 


\section{Results}

137 patients with Neurogenic TOS underwent robotic transthoracic resection of the offending portion of the first rib with scalenectomy and disarticulation of the costo-sternal joint. There were 47 men and 90 women. Mean age was $34 \pm 9.5$ years.

In all patients localizing and specific diagnosis for the presence of upper extremity neurologic exam was ruled out. Nerve conduction tests were negative for nerve compression in all patients. Based on these parameters all patients were classified as “Disputed” Neurogenic TOS. Surgery was offered after the patient's symptoms were determined to be refractory to physical therapy. Preoperatively, MRA with maneuvers showed extrinsic compression of the subclavian vein at its junction with the innominate vein in all patients.

The "offending portion of the first rib" which included the rib medial to the subclavian artery and the costo-sternal joint was removed en bloc. In all patients, a bony protuberance resulted in a wider more pronounced costo-sternal joint (Figure 2). Operative time was 93 minutes \pm 10.3 minutes. There were no intraoperative complications. There was no injury to the subclavian vessels during the dissection. There were no neurovascular complications. There was no 30 or 90 day mortality.

Hospital stay ranged from 3 to 6 days with a median hospitalization of 3 days. Prolonged hospitalization was due to the management of pain and chest tube drainage.

Quick DASH Scores (Mean \pm SEM) decreased from $60.3+/ 2.1$ preoperatively to $5 \pm 2.3$ in the immediate postoperative period, and $3.5+/ 1.1$ at 6 months. $(\mathrm{P}<$ $0.01)$ Immediate relief of symptoms was seen in all patients (100\%). Complete relief of symptoms was seen at 6 months in 133/137 (97\%). Persistent paresthesia was seen in $4 / 137(3 \%)$ at 6 months. Of interest, these patients were among the oldest in the patient population and had experienced upper extremity pain for greater than 10 years.

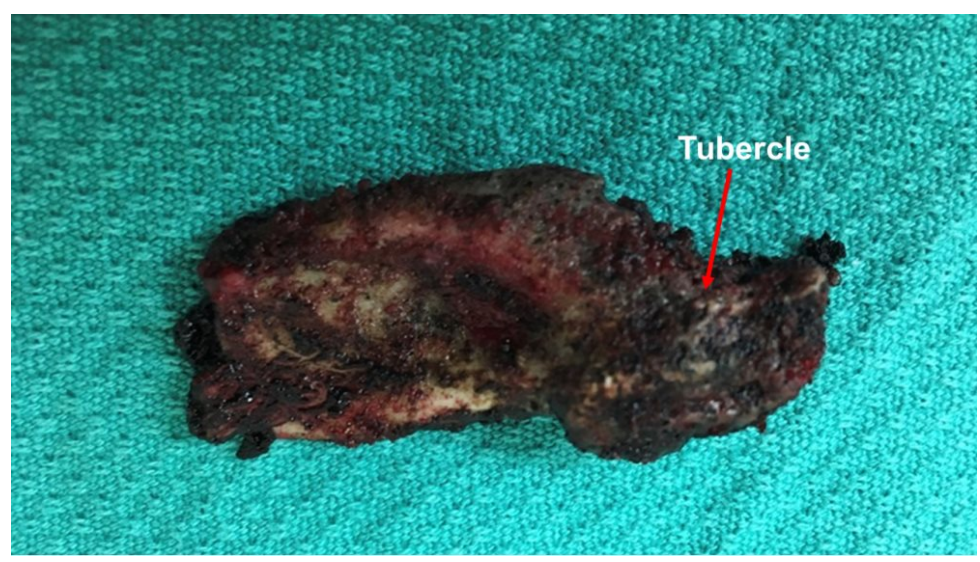

Figure 2. The resected specimen of the "offending" portion of the first rib from a patient with Disputed Neurogenic TOS syndrome showing the abnormal bony tubercle on the medial aspect of the first rib (arrow) that compresses the subclavian vein at the subclavian-innominate junction. 
On postoperative MRA, there was relief of extrinsic compression of the subclavian vein in all patients.

Follow-up was complete in all patients. Median Follow up was 6 months. At the time of follow up, $98.6 \%$ of patients had complete relief of symptoms.

\section{Discussion}

Since Peet's classification of TOS in 1956, conventionally TOS has been thought to represent a group of diverse disorders that result in compression of the neurovascular bundle exiting the thoracic outlet. Compression of this area has been thought to result in a constellation of distinct symptoms, which can include upper extremity pallor, paresthesia, weakness, muscle atrophy, pain, and swelling [11] [12]. Until recently, classification of TOS has been based on symptoms, rather than the underlying pathology, with the subgroups consisting of neurogenic (NTOS), venous (VTOS or PSS), and arterial (ATOS). Neurogenic TOS accounts for over $95 \%$ of the cases, followed by venous $(3 \%-5 \%)$ and arterial $(1 \%-2 \%)$. Neurogenic TOS (NTOS) has been further divided into True NTOS (TNTOS) and Disputed NTOS (DNTOS), with DNTOS reportedly representing 95\% - 99\% of all neurogenic cases [12]. The symptoms of TNTOS and DNTOS are largely the same, though objective findings from motor nerve conduction studies and needle electromyography are notably absent in the DNTOS.

Examination of the medial aspect of the resected first ribs in patients with $\mathrm{Pa}$ get-Schroetter syndrome has demonstrated the presence of a congenitally malformed bony tubercle that forms a tighter and wider joint at the junction of the first rib and the sternum, the costo-sternal joint [13]. It has been observed that the wider and less mobile cost-sternal joint "locks" the medial aspect of the first rib into place and results in extrinsic compression of the boney tubercle onto the subclavian vein at its junction with the innominate vein. Therefore, the abnormal medial aspect of the first rib compresses the subclavian vein at the "thoracic outlet." This abnormal tubercle on the medial aspect of the first rib can be seen on 3-D reconstruction of computerized axial tomograms in patients with PSS (Figure 3). In addition, the extrinsic compression of the subclavian vein by the tubercle at the medial aspect of the first rib can be demonstrated on dynamic magnetic resonance angiography (MRA) (Figure 4). Furthermore, these studies clearly demonstrate that the subclavian vein compression increases with elevation of the arm above the shoulder. In patients with Paget-Schroetter syndrome, Gharagozloo et al. have demonstrated that disarticulation of the costo-sternal joint and resection of the offending portion of the first rib (portion of the rib medial to the subclavian artery) result in decompression of the subclavian vein and excellent long term patency of the subclavian vein [8].

A retrospective study comparing normal human cadaver ribs to the resected first ribs from patients with DNTOS has demonstrated a boney tubercle similar to that seen in patients with PSS at the medial aspect of the first rib which forms an abnormal costo-sternal joint. It has been proposed that is the pathologic entity 


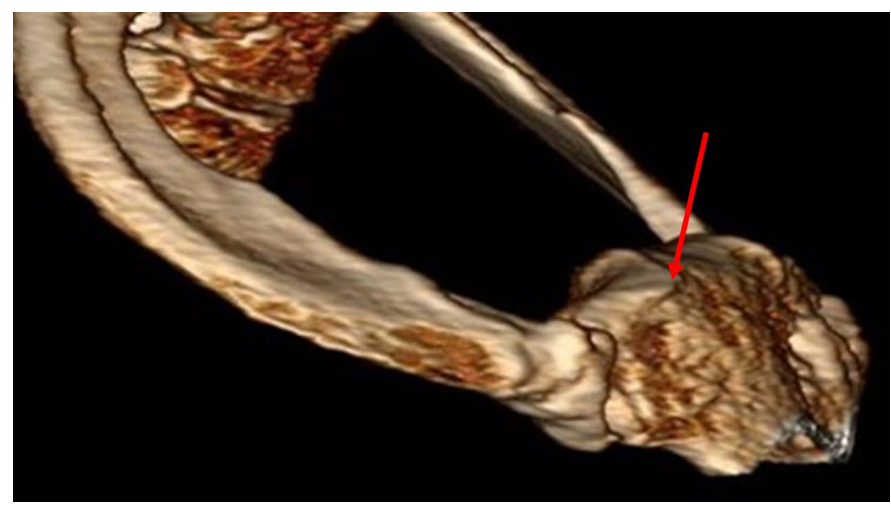

Figure 3. 3-D reconstruction of computerized axial tomograms in patients with Paget-Schroetter syndrome demonstrates the wider and less mobile cost-sternal joint (arrow) that "locks" the medial aspect of the first rib into place and results in extrinsic compression of the bony tubercle onto the subclavian vein at its junction with the innominate vein.

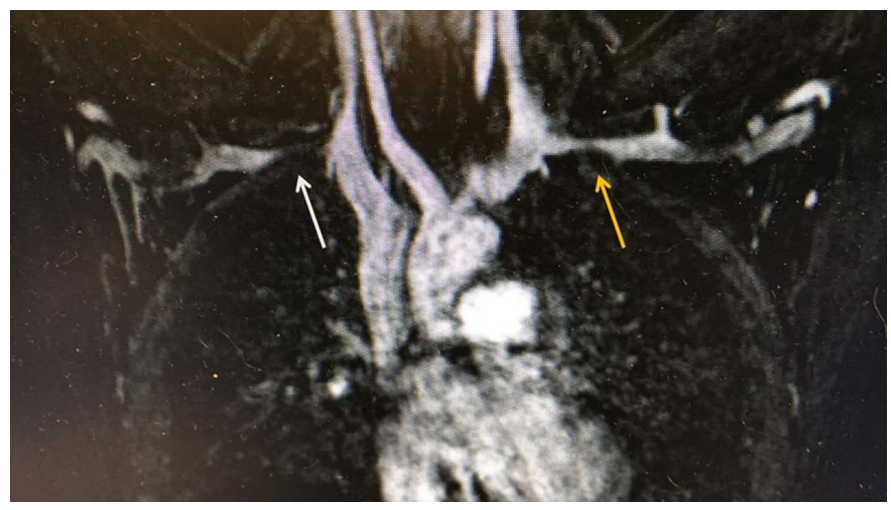

Figure 4. Magnetic resonance angiogram (MRA) with elevation of the left upper extremity shows compression of the subclavian vein by the abnormal bony tubercle in a patient with Paget-Schroetter Syndrome.

which causes extrinsic compression of the subclavian vein and that the neurologic symptoms may be due to venous compression and the resultant venous ischemia of the nerves in the upper extremity [14]. Furthermore, it has been surmised that without the benefit of the sophisticated modern imaging, and relying only on intraoperative observations, it is likely that historically surgeons have erroneously referred to this tubercle as the hypertrophied costoclavicular ligament and the hypertrophied scaleneus anticus tubercle. This hypothesis is supported by the fact that the upper extremity is fed by a single artery and a single vein as an "end organ". In such a setting, studies have demonstrated that the blood-nerve barrier in the nerve root was more easily broken by venous congestion than by arterial ischemia. Venous congestion may be an essential factor precipitating circulatory disturbance in nerve roots and inducing neurogenic intermittent claudication [14]. This phenomenon is illustrated by the feeling of a leg "falling asleep" or pain and paresthesia which is experienced in the lower ex- 
tremity after crossing the leg for a prolonged period of time. Of interest, patients with DNTOS liken their symptoms to their arms "falling asleep".

The similarity of the pathologic findings in the medial aspect of the resected first ribs and the cost-asternal joint in patients with DTNOS and PSS, may suggest that compression of the subclavian vein by the abnormal boney tubercle and the costo-sternal joint is the common pathologic pathway. Compression of the vein results in venous hypertension in the upper extremity and resultant neurologic symptoms. With prolonged compression of the subclavian-innominate junction, the vein clots giving rise to Paget-Schroetter syndrome. Therefore, patients who have been previously classified as neurogenic and venous TOS may represent a variable symptomatic presentation of the same pathologic entity.

The first rib is most commonly removed using a Transaxillary or Supraclavicular approaches. In general, the extrathoracic approaches to the first rib have been hampered by the potential of neurovascular complications and incomplete resection of the medial portion of the first rib. Thoracoscopic first rib resection has been proposed as a minimally invasive approach to the first rib that could potentially obviate retraction of the neurovascular structures necessitated by the extra thoracic approaches. However, although this procedure has been based on sound reasoning, it is limited by shortcomings of the conventional endoscopic instruments and two-dimensional visualization. Robotic surgical systems allow for high-definition, magnified, three-dimensional visualization of the operative field, are associated with accurate instrument maneuverability in a confined space, and may overcome the potential shortcomings of the conventional thoracoscopic approach. Robotic first rib resection has been shown to be feasible and is associated with excellent results [8].

In this report from a 10 year period, 137 patients with DNTOS underwent robotic resection of the medial aspect of the first rib with disarticulation of the costo-sternal joint without neurovascular complications. As has been reported previously, due to the transthoracic approach, patients experienced greater pain and drainage of the chest which resulted in a longer hospital stay [8].

Preoperative MRA showed extrinsic compression of the subclavian-innominate junction at rest and increase of the compression with elevation of the arm in all patients (Figure 5). Postoperative MRA showed relief of the extrinsic compression of the subclavian-innominate junction at rest and with arm elevation (Figure 6). Intraoperatively the subclavian-innominate junction was decompressed with the disarticulation of the costo-sternal joint (Figure 7). This finding correlated with complete symptom relief in $97 \%$ of patients.

Following the procedure, all patients experienced relief of symptoms. However "Complete" symptom relief was reported by $97 \%$ of patients at 6 months. $3 \%$ of patients experienced occasional paresthesia even though their neurologic symptoms were dramatically improved. These patients were older and had symptoms for a longer period of time. It is possible that their symptoms would continue to improve. However, assessment of this hypothesis would require longer follow-up and may be the subject of a future study. 


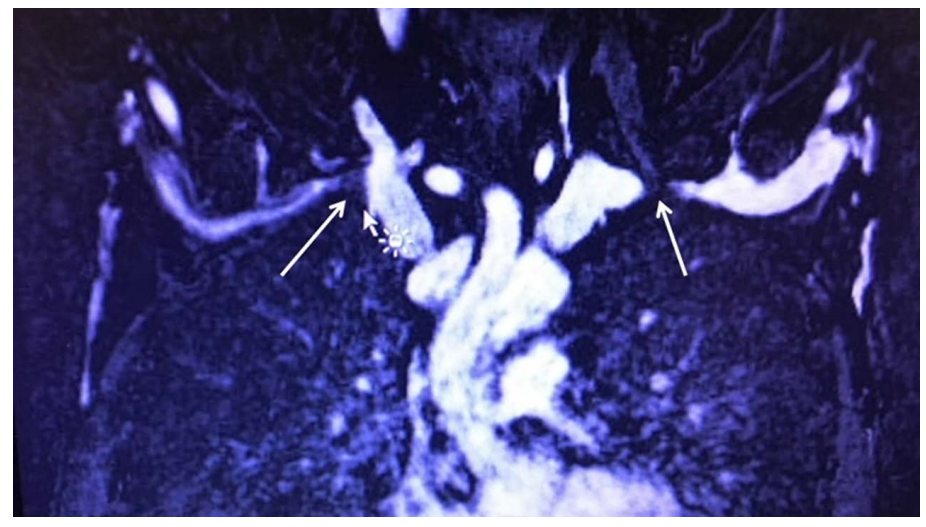

Figure 5. Preoperative Magnetic resonance angiogram (MRA) in a patient with Disputed Neurogenic TOS. With elevation of the arms, there is bilateral compression (arrows) of the subclavian innominate vein junction.

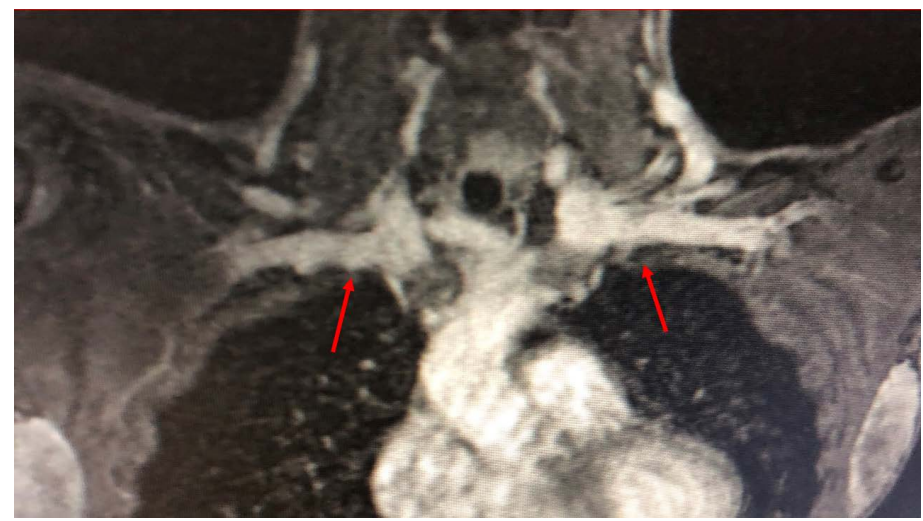

Figure 6. Postoperative Magnetic resonance angiogram (MRA) in a patient with Disputed Neurogenic TOS following bilateral staged robotic resection of the offending portion of the first rib and disarticulation of the costo-sternal joint. The extrinsic compression at the subclavian-innominate junction is no longer present (arrows).

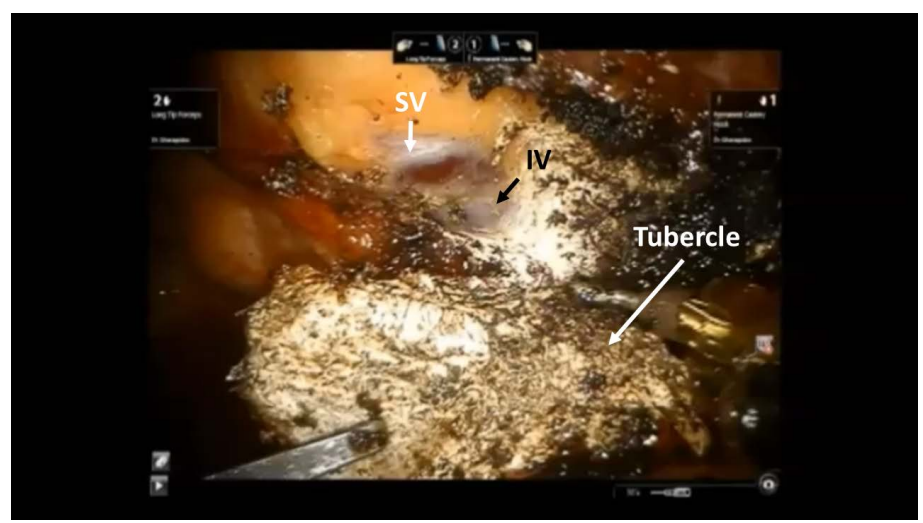

Figure 7. Intraoperative photograph during the robotic resection of the medial right first rib in a patient with Disputed Neurogenic TOS. The abnormal boney tubercle at the costo-steral joint results in compression of the subclavian vein (SV) at its junction with the innominate vein (IV). 


\section{Study Limitations}

The following limitations of this study should be considered before drawing definitive conclusions.

The study was retrospective and represented a highly selected group of patients.

Undoubtedly the use of robotic technology adds more ports, resulting in greater short term postoperative morbidity, longer hospitalization and greater cost. These shortcomings may be obviated by the low incidence of complications and the excellent postoperative symptom relief.

\section{Conclusion}

Robotic resection of the medial aspect of the first rib with disarticulation of the costo-sternal joint is associated with excellent relief of neurologic symptoms in patients with Neurogenic Thoracic Outlet Syndrome.

\section{Conflicts of Interest}

The authors declare no conflicts of interest regarding the publication of this paper.

\section{References}

[1] Peet, R.M. (1956) Thoracic-Outlet Syndrome: Evaluation of a Therapeutic Exercise Program. Proceedings of the Staff Meetings of Mayo Clinic, 31, 281-287.

[2] Freischlag, J. and Orion, K. (2014) Understanding Thoracic Outlet Syndrome. Scientifca (Cairo), 2014, Article ID: 248163. https://doi.org/10.1155/2014/248163

[3] Falconer, M.A. and Li, F.W. (1962) Resection of the First Rib in Costoclavicular Compression of the Brachial Plexus. Lancet, 279, 59-63.

https://doi.org/10.1016/S0140-6736(62)91716-6

[4] Gharagozloo, F., Werden, S., Meyer, M., CST-FA, A.B., Hallman-Quirk, H., Ware, A., et al. (2022) Historic Basis for the New Developments in the Diagnosis and Treatment of Thoracic Outlet Syndrome (TOS). Clinc Surg J, 3, 1-16.

[5] Weaver, M. and Lum, Y. (2017) New Diagnostic and Treatment Modalities for Neurogenic Thoracic Outlet Syndrome. Diagnostics, 7, 28. https://doi.org/10.3390/diagnostics7020028

[6] Hwang, J., Min, B.J., Jo, W.-M. and Shin, J.S. (2017) Video-Assisted Thoracoscopic Surgery for Intrathoracic First Rib Resection in Thoracic Outlet Syndrome. Journal of Thoracic Disease, 9, 2022-2028. https://doi.org/10.21037/jtd.2017.06.122

[7] Gharagozloo, F., Meyer, M., Tempesta, B., et al. (2012) Robotic En Bloc First-Rib Resection for Paget-Schroetter Disease, a Form of Thoracic Outlet Syndrome: Technique and Initial Results. Innovations, 7, 39-44. https://doi.org/10.1097/imi.0b013e3182542ab3

[8] Gharagozloo, F., Meyer, M., Tempesta, B., et al. (2019) Robotic Transthoracic First Rib Resection for Paget-Schroetter Syndrome. European Journal of Cardiothoracic Surgery, 55, 434-439. https://doi.org/10.1093/ejcts/ezy275

[9] Gummesson, C., Ward, M.M. and Atroshi, I. (2006) The Shortened Disabilities of the Arm, Shoulder and Hand Questionnaire (Quick DASH): Validity and Reliability 
Based on Responses within the Full-Length DASH. BMC Musculoskeletal Disorders, 7, Article No. 44. https://doi.org/10.1186/1471-2474-7-44

[10] Matheson, L.N., Melhorn, M., Mayer, T.G., et al. (2006) Reliability of a Visual Ana$\log$ Version of the Quick DASH. The Journal of Bone \& Joint Surgery, 88, 1782-1787. https://doi.org/10.2106/JBJS.F.00406

[11] Stewman, C., Vitanzo, P.C. and Harwood, M.I. (2014) Neurologic Thoracic Outlet Syndrome: Summarizing a Complex History and Evolution. Current Sports Medicine Reports, 13, 100-106. https://doi.org/10.1249/JSR.0000000000000038

[12] Ferrante, M.A. and Ferrante, N.D. (2017) The Thoracic Outlet Syndromes: Part 2. The Arterial, Venous, Neurovascular, and Disputed Thoracic Outlet Syndromes. Muscle Nerve, 56, 663-673. https://doi.org/10.1002/mus.25535

[13] Gharagozloo, F., Meyer, M., Tempesta, B., et al. (2012) Proposed Pathogenesis of Paget-Schroetter Disease: Impingement of the Subclavian Vein by a Congenitally Malformed Boney Tubercle on the First Rib. Journal of Clinical Pathology, 65, 262-266. https://doi.org/10.1136/jclinpath-2011-200479

[14] Kobayashi, S., Takeno, K., Miyazaki, T., et al. (2008) Effects of Arterial Ischemia and Venous Congestion on the Lumbar Nerve Root in Dogs. Journal of Orthopaedic Research, 26, 1533-1540. https://doi.org/10.1002/jor.20696 